\title{
Kernos
}

Revue internationale et pluridisciplinaire de religion grecque antique

$21 \mid 2008$

Varia

\section{Laurent BRICAULT, Isis, Dame des flots}

\section{Richard Veymiers}

\section{(2) OpenEdition \\ Journals}

\section{Édition électronique}

URL : https://journals.openedition.org/kernos/1692

DOI : 10.4000/kernos. 1692

ISSN : 2034-7871

Éditeur

Centre international d'étude de la religion grecque antique

Édition imprimée

Date de publication : 1 janvier 2008

Pagination : 335-337

ISSN : 0776-3824

Référence électronique

Richard Veymiers, "Laurent bricault, Isis, Dame des flots », Kernos [En ligne], 21 | 2008, mis en ligne le 15 septembre 2011, consulté le 24 août 2022. URL : http://journals.openedition.org/kernos/1692 ;

DOI : https://doi.org/10.4000/kernos. 1692 
vant une panoplie, non aux Dionysies, mais aux concours organisés lors des Héracleia ( $c f$. la Stèle des Braves de Thasos). Je me demande donc si ces groupes marginaux pointés par l'A. comme étant spécifiques aux fêtes dionysiaques le sont vraiment et si le sujet n'aurait pas requis d'élargir la perspective, en sortant du monde trop étroitement dionysiaque.

Une conception de la politique et une certaine vision du monde sont les deux faciès de l'identité athénienne qui s'expriment ainsi à travers ces quatre fêtes. Un même système de valeurs constitue le noyau de ces quatre fêtes qui peuvent ainsi se corréler l'une l'autre. La référence au vin est l'un des dénominateurs communs aux Anthestéries, aux Lénéennes et aux Dionysies aux champs, mais elle n'apparait pas clairement dans les Grandes Dionysies. D'autre part, toutes ces fêtes n'accueillant pas les mêmes marginaux, l'A. montre qu'un système de compensations devait réguler la participation aux fêtes de ces groupes. Ainsi, si les Lénéennes ne comportent pas d'étrangers, elles admettent la participation exceptionnelle de métèques dans la composition des chœurs; d'autre part, les étrangers participent aux Grandes Dionysies, mais les métèques n'y sont pas admis dans les chœurs. L'ouverture sur les étrangers, les esclaves, le monde extérieur est maximale dans les Grandes Dionysies, mineure dans les Lénéennes, mais est compensée par un rôle exceptionnel donné aux métèques et aux étrangers présents à Athènes. L'étude des fêtes et des systèmes rituels qui les sous-tendent (dont la base commune est la suspension de l'ordre) montre qu'à l'époque classique les rites dionysiaques sont parfaitement intégrés dans les structures politiques, religieuses et sociales de la cité grecque.

L'éclairage très particulier que donne l'A. à ces quatre fêtes dionysiaques n'épuise pas, à mon avis, le sujet complexe et plein de zones d'ombre par manque de témoignages, des fêtes dionysiaques. L'ouvrage ne prend pas en considération la documentation figurée et sans doute peut-on le regretter car les images, mêmes bien connues, auraient probablement pu apporter des informations complémentaires à son propos. La délicate question des Anthestéries/Lénéennes, de l'ouverture des jarres et des images montrant des rituels féminins devant les jarres aurait mérité une nouvelle approche iconographique. Il n’en reste pas moins qu'il apporte un éclairage nuancé sur la société athénienne de l'époque classique et qu'il constitue une somme d'informations qui marquera les études dionysiaques.

Isabelle TASSIGNON (FUNDP - Namur)

Laurent BriCAUlT, Isis, Dame des flots, Liège, CIPL, 2006. 1 vol. $18 \times 26 \mathrm{~cm}$, 244 p. 3 cartes, 80 fig. (Aegyptiaca Leodiensia, 7).

Cette enquête met en avant en sept chapitres les liens qui unissent Isis, et dans une moindre mesure Sarapis, à l'élément marin. Usant de nombreux documents, tant textuels qu'iconographiques, et souvent méconnus, sinon inédits, L. Bricault présente avec clarté les divers et délicats problèmes que soulève cette importante prérogative de la déesse. Le titre choisi témoigne déjà de la précision et de l'ingéniosité dont fait preuve l'A. tout au long de son analyse. Isis est bien une Dame des flots, mais pas, à proprement parler, une déesse marine, puisqu'elle ne vit pas dans la mer.

Il est d'abord question des origines de cet aspect d'Isis (p. 13-36). Dans l'Égypte pharaonique, seuls Amon et Hathor paraissent avoir été parfois considérés comme des maitres des flots. Isis n'a alors que des liens ténus avec l'élément aquatique, qu'il soit fluvial ou maritime. Plutôt que les Égyptiens, ce sont les Phéniciens de Byblos, voire les Grecs de Naucratis, qui pourraient avoir indirectement joué un rôle dans l'acceptation d'Isis en tant que déesse maritime. Ce trait n'apparait toutefois réellement qu'au début du $\mathrm{III}^{\mathrm{e}}$ siècle av. J.C. sous l'influence d'Arsinoé II et de son amiral, Callicratès de Samos. Une fresque de 
Nymphaion, en Crimée, montrant un navire inscrit du nom "Isis », en serait la première attestation. C'est, semble-t-il, d'Arsinoé II, assimilée à Aphrodite Euploia, qu'Isis hérite sa qualité de Dame des flots.

Cette prérogative nouvelle, purement grecque, est ensuite théorisée dans le récit arétalogique, probablement conçu à Memphis à la fin du $\mathrm{III}^{\mathrm{e}}$ ou au début du $\mathrm{II}^{\mathrm{e}}$ siècle av. J.-C., avant d'être reprise dans l'un des hymnes d'Isidôros à Medinet Madi et dans la litanie oxyrhynchite (p. 37-42). L'un des traits les plus originaux diffusés par l'Arétalogie parait bien être l'invention des activités maritimes. Cette arétè d'Isis amènera à voir en elle l'inventrice de la voile et à la créditer d'une géniale initiative : c'est de son manteau qu'elle fit une voile.

Cette évolution théologique se traduit par des images d'Isis tenant une voile gonflée, un type qui apparaît à Byblos sous le règne d'Antiochos IV avant de se diffuser sous diverses variantes dans le monde méditerranéen (p. 43-80). En l'absence de traits spécifiquement isiaques, comme un sistre et/ou un basileion, il n'est pas toujours assuré que la déesse «à la voile » soit une Isis. L'identification doit alors s'opérer par comparaison ou contextualisation. Une série de documents, mêlant aspects maritime et frugifère, semblent mettre en relation Isis avec l'Annone. La flotte frumentaire d'Alexandrie y apparaît « sous la triple protection de Déméter, déesse des récoltes, de Sarapis, dieu de la végétation et protecteur des marins, enfin d'Isis, à la fois dispensatrice des richesses (elle tient la cornucopia), garante du bon acheminement de la cargaison (elle tient la voile et/ou le gouvernail), et bonne fortune (elle est de fait assimilée à Tychè) ». Notre Dame des flots a donc pu être aussi bien représentée sous l'aspect d'Isis à la voile que sous celui d'Isis au gouvernail et éventuellement à la cornucopia (p. 80-86). Quant à l'existence, longtemps débattue, de représentations statuaires d'Isis «à la voile» (p. 86-99), elle semble aujourd'hui confirmée par une statue découverte près de l'Iseion de Messène.

Plusieurs qualificatifs ont pu servir à désigner l'Isis, Dame des flots (p. 101-112). On ne peut toutefois les utiliser comme autant de synonymes. L'épithète Euploia, surtout employée à l'époque hellénistique, comme celle de Pelagia, qui fleurit plutôt à l'époque impériale, recouvrent généralement une Isis assurant une bonne navigation. La polysémique Sôteira met l'accent sur la protection qu'elle apporte aux marins après avoir écouté leurs prières. L'épithète Pharia, la plus fréquente, se rapporte sans doute à l'Isis alexandrine, devenue protectrice de la flotte frumentaire qui transporte le blé d'Égypte en Italie. Enfin, Isis est encore dite Kubernètis, " pilote », et Ormistria, « Notre-Dame-du-Havre ».

Isis Pelagia a pu bénéficier de lieux de culte propres, ainsi que l'attestent le texte de Pausanias sur Corinthe et une inscription funéraire de Rome (p. 113-123). Contrairement aux autres qualificatifs, Pelagia est donc bien une épiclèse de la déesse, non une simple épithète. En tant que patronne des flots, Isis avait droit à des offrandes particulières (p. 123134). Les marins pouvaient lui offrir, avant leur départ, de petites ancres ou des représentations d'animaux marins, et, au terme d'une traversée heureuse, des peintures, parfois à la suite de l'acquittement d'un vœu exaucé. Les moins fortunés devaient se servir d'amulettes pour invoquer sa protection. Le cas des lampes naviformes, portant un type isiaque, est délicat. Quel que soit leur rôle (liturgique, votif, funéraire, voire simplement fonctionnel et décoratif), il est difficile de considérer que leurs propriétaires, ou leurs dédicants, n’aient pas eu un quelconque rapport avec le milieu maritime. Deux grandes fêtes honorent dans le calendrier romain Isis, dame de la mer (p. 134-154). Remontant au Ier s. av. J.-C., voire plus tôt, les Ploiaphesia ou le Navigium Isidis marquent, à la date du 5 mars, la réouverture solennelle de la navigation en Méditerranée. Au cours de cette célébration, décrite au livre XI des Métamorphoses d'Apulée, une longue procession précède le lancement du navire "Isis", auquel participent des navarques, ceux des fidèles qui forment l'équipage, sous la direction d'un triéraque, l'organisateur de la cérémonie. La seconde festivité, le Sacrum Phariae du 25 
avril, semble être mise en relation avec l'arrivée à Ostie de la flotte frumentaire, placée sous la sauvegarde d'Isis et de Sarapis.

Sous l'influence d'Isis, Sarapis devient à l'époque impériale, sinon avant, lui aussi, un Pelagius (p. 155-167). La diffusion de l'histoire du songe de Ptolémée, rapportée par Tacite et Plutarque, a pu jouer un rôle dans cette évolution. Toutefois, Sarapis assume rarement seul son rôle marin et réserve son action aux domaines plus strictement économique et politicomilitaire. Protecteur des marins, il apparait, de même qu'Isis, comme parasème de nombre de navires (p. 168-170).

Cette popularité ne s'est pas démentie jusqu'à la fin du IVe siècle, ainsi qu'en témoignent les monnaies romaines frappées à l'occasion des Vota publica (p. 171-172). À en croire Végèce et Jean Le Lydien, le Navigium Isidis était encore célébré au Ve, voire au VIe siècle. La maitrise d'Isis sur les flots n'a jamais ensuite été véritablement oubliée (p. 172-176). On en trouve, par exemple, écho sur les nouvelles armoiries de Paris en 1811.

Dans sa conclusion (p. 177-179), l'A. récapitule les principaux points qui se dégagent de son enquête. Un corps d'index (p. 181-190) ainsi qu'une riche bibliographie (p. 191-238) complètent le volume. Nul doute que cette très belle synthèse des aspects marins d'Isis et Sérapis deviendra un modèle pour tous ceux qui voudront s'engager dans les études isiaques. On ne peut qu'en remercier l'A. et attendre avec impatience sa prochaine enquête consacrée aux aspects guérisseurs du couple isiaque.

Richard VEYMIERS

(F.R.S.-FNRS - Université de Liège)

Nadine Deshours, Les Mystères d'Andania. Étude d'épigraphie et d'bistoire religieuse, Bordeaux, Diffusion De Boccard, 2006. 1 vol. 16,5 × 22,5 cm, 278 p. (Ausonius. Scripta Antiqua, 16). ISBN : 2-910023-72-9.

Les mystères d'Andania, en Messénie, sont connus par une double tradition documentaire : des inscriptions et le texte de Pausanias au livre IV de sa Périégèse. Ce dernier retrace longuement les vicissitudes du peuple messénien soumis par les Spartiates, en mettant particulièrement en évidence l'enfouissement par le héros local Aristomène d'une sorte de «talisman » nécessaire au retour des Messéniens dans leur patrie. L'objet en question serait une hydrie de bronze contenant des lamelles d'étain consignant la teletè des Grandes Déesses, Déméter et sa fille. Elles auraient été honorées de longue date par un culte à mystères d'origine éleusinienne. Toujours selon Pausanias, cette hydrie aurait été redécouverte au moment de la fondation de Messène par Épaminondas et le culte réactivé dans l'alsos Karnasion dans la campagne messénienne. Le visiteur affirme d'ailleurs avoir vu le vase lors de sa visite de l'alsos.

Parmi les inscriptions qui forment l'autre volet documentaire, la plus justement célèbre est un long règlement de 194 lignes, exceptionnel par les détails qu'il livre pour l'organisation de mystères dans l'alsos Karneiasion et par son excellent état de conservation. Après un status quaestionis bien informé, c'est sur ce document que s'ouvre l'ouvrage de Nadine Deshours qui en produit une édition et une traduction en français, après celles qu'avait données Paul Foucart en 1876 dans le Voyage archéologique en Grèce et en Asie Mineure. Suivent alors deux parties d'une centaine de pages chacune, la première qui est un commentaire thématique de l'inscription, la seconde qui inscrit le document sur l'arrière-plan auquel il appartient, à savoir l'histoire mouvementée de la Messénie telle que notre seule source circonstanciée la retrace, à savoir la Périégèse de Pausanias. 